\title{
Pneumococcal serotypes in adult non-invasive and invasive pneumonia in relation to child contact and child vaccination status
}

\author{
Chamira Rodrigo, ${ }^{1}$ Thomas Bewick, ${ }^{1}$ Carmen Sheppard, ${ }^{2}$ Sonia Greenwood, ${ }^{1}$ \\ Vanessa MacGregor, ${ }^{3}$ Caroline Trotter, ${ }^{4}$ Mary Slack, ${ }^{2}$ Robert George, ${ }^{2}$ Wei Shen Lim ${ }^{1}$
}

\section{- Additional material is published online only. To view please visit the journal online (http://dx.doi.org/10.1136/ thoraxjnl-2013-203987) \\ 1 Department of Respiratory Medicine, City Hospital Campus, Nottingham University Hospitals NHS Trust, Nottingham, UK \\ ${ }^{2}$ Respiratory and Systemic Infection Laboratory, Public Health England, Microbiology Services Division, London, UK ${ }^{3}$ Public Health England East Midlands, Derbyshire and Nottinghamshire Health Protection Team, Institute of Population Health, Nottingham City Hospital, Nottingham, UK ${ }^{4}$ Disease Dynamics Unit, Department of Veterinary Medicine, University of Cambridge, Cambridge, UK}

\section{Correspondence to} Dr C Rodrigo, Department of Respiratory Medicine, David Evans Building, City Hospital Campus, Nottingham University Hospitals NHS Trust, Hucknall Road, Nottingham NG51PB, UK;

chamira@doctors.org.uk

Received 8 June 2013 Revised 27 August 2013 Accepted 5 September 2013 Published Online First 18 September 2013
To cite: Rodrigo C, Bewick T, Sheppard C, et al. Thorax 2014;69:168-173.

\section{ABSTRACT}

Background On a population level, pneumococcal conjugate vaccination in children has reduced the incidence of vaccine-type disease in all age groups, including older adults. Few individual level studies have been performed describing the pneumococcal serotypes associated with adult community acquired pneumonia (CAP) and quantifying associations with child contact and child vaccination status.

Methods Pneumococcal serotypes were determined using a validated multiplex immunoassay (Bio-Plex) in a large prospective cohort of adults hospitalised with CAP. Child ( $<16$ years old) contact history and child pneumococcal vaccination status were obtained from patients and public health records, respectively.

Results Of 1130 participants, 329 (29.1\%) reported child contact, and pneumococcal infection was identified in 410 (36.3\%). Pneumococcal CAP was commoner in adults with child contact (148/329 (45.0\%) vs $262 / 801$ (32.7\%); adjusted OR 1.63, Cl 1.25 to $2.14 ; \mathrm{p}<0.001)$. A serotype was determined in 263 of $410(64.1 \%)$ adults with pneumococcal CAP; $112(42.6 \%)$ reported child contact, 38 (33.9\%) with a vaccinated child. Adults in contact with a vaccinated child were significantly less likely to have vaccine-type CAP compared with adults in contact with an unvaccinated child (6 of $38(15.8 \%$ ) vs 25 of $74(33.8 \%)$, respectively; OR $0.37,95 \% \mathrm{Cl} 0.14$ to $0.99 ; p=0.044)$.

Conclusions Pneumococcal aetiology in adult CAP is independently associated with child contact and implicated serotypes are influenced by child vaccination status. This is the first study to demonstrate these associations at an individual rather than population level; it affirms that 'herd protection' from childhood vaccination extends beyond adult invasive disease to pneumococcal CAP.

\section{BACKGROUND}

Community acquired pneumonia (CAP) accounts for over 100000 admissions in the $\mathrm{UK}^{1}$ with an associated mortality of $6-24 \% .^{2}{ }^{3}$ Streptococcus pneumoniae remains the commonest cause of CAP in hospitalised adults. ${ }^{45}$

Nasopharyngeal colonisation is integral to the pathogenesis of pneumococcal disease. ${ }^{6}$ Colonisation rates peak in early childhood (43-52\% in children under 4 years, $21 \%$ in $5-17$ years) and decline to around $5-8 \%$ in adults over 18 years in developed countries. ${ }^{7}$ Following establishment of colonisation, horizontal transmission of

\section{Key messages}

What is the key question?

- In adults admitted to hospital with community-acquired pneumonia, does contact with a child and the pneumococcal conjugate vaccination status of the child influence the likelihood of pneumococcal pneumonia and the implicated serotype?

\section{What is the bottom line?}

- Close contact with a child increases the odds of pneumonia due to Streptococcus pneumoniae in adults; contact with a pneumococcal conjugate vaccinated child is associated with the development of non-vaccine-serotype pneumococcal pneumonia in adults.

\section{Why read on?}

- This is the first study to demonstrate the effects of 'herd protection' from childhood pneumococcal conjugate vaccination at an individual level in an adult cohort of predominantly non-invasive pneumococcal disease.

pneumococci occurs through contact with the secretions of these carrier individuals. Studies of nasopharyngeal culture from healthy children and their families have demonstrated transmission of homologous serotypes to other siblings and adults within the same household. ${ }^{8}$ Day-care attendance and the presence of pre-school age siblings are risk factors for the development of invasive pneumococcal disease (IPD) in children. ${ }^{10}$ Child contact has been shown to be a risk factor for the development of IPD in adults. ${ }^{11} 12$

The seven-valent pneumococcal conjugate vaccine (PCV7) was introduced into the childhood immunisation programme in September 2006 in the UK, followed by a 13-valent vaccine (PCV13) in April 2010. Since introduction of childhood pneumococcal vaccination, overall rates of IPD have declined in children ${ }^{13}{ }^{14}$ with parallel reductions in vaccine-type IPD rates in adults, most likely due to herd protection. ${ }^{13-16}$ The effect of this herd protection in reducing rates of non-invasive disease in adults has been more difficult to demonstrate. 1718 
The relationships between pneumococcal serotype, child contact and non-invasive pneumococcal CAP in adults have not been investigated in the past. The aim of this study was to investigate the association of child contact and child pneumococcal vaccination status with the occurrence of adult pneumococcal pneumonia.

\section{METHODS}

An observational prospective cohort study was conducted at two large UK teaching hospitals, from September 2008 to September 2011. Consecutive adult patients (aged over 16) admitted with CAP were recruited. CAP was defined as symptoms of lower respiratory tract infection (at least one of increasing breathlessness, cough, sputum or fever) with new infiltrates on chest radiography and treated by the admitting team as for CAP. Patients were excluded in the event of post-obstruction pneumonia due to lung cancer, discharge from hospital in the preceding 10 days or had active tuberculosis. Study investigators identified patients from the acute admission units and wards on a daily basis. Patients were enrolled following informed consent. A standardised proforma was used to collect patient demographics, clinical information, radiological findings, microbiological investigations and outcome measures. Data regarding 30-day mortality were retrieved from the local hospital patient information database. Information regarding child contact was obtained from the patient or a relative during the interview. Child contact was defined as living with a child ( $\leq 16$ years) within the same household, contact with a child for more than $8 \mathrm{~h}$ in the 4 weeks prior to illness or an occupation involving full-time contact with children (eg, teaching). As there is no standard definition of child contact in relation to pneumococcal disease, the definition used in this study is similar to that used for public health purposes in the context of meningococcal disease where nasopharyngeal carriage is also important. ${ }^{19}$ If child contact was present, demographic details of the child were obtained by recall and details of vaccination were obtained either from primary care vaccination records held by the General Practitioner or from the local Child Health Information System.

Urine samples were obtained from patients on admission and sent to the Nottingham University Hospitals Department of Clinical Microbiology, a regional Public Health England (PHE) laboratory. Samples were tested using the BinaxNOW (Alere) immunochromatographic test kit according to the manufacturer's instructions. Following this, samples were kept in frozen storage and transported in batches to the Respiratory and Systemic Infection Laboratory (RSIL) at the Centre for Infections (CfI) at PHE Colindale, London for pneumococcal serotyping. This was carried out using a multiplex immunoassay (Bio-Plex; Bio-Rad) capable of detecting 14 pneumococcal serotypes $(1,3,4,5,6 \mathrm{~A} / \mathrm{C}, 6 \mathrm{~B}, 7 \mathrm{~F} / \mathrm{A}, 8,9 \mathrm{~V}, 14,18,19 \mathrm{~A}, 19 \mathrm{~F}$ and $23 \mathrm{~F}$ ) with $79 \%$ sensitivity. ${ }^{20}$ Serotype was also determined in patients with bacteraemia using slide agglutination using the full set of serotyping latex and factor serum, available from Statens Serum Institut Copenhagen. This method is capable of determining all serotypes of pneumococci other than the newly described serotype 11E. All adults included in the cohort $(n=1130)$ had BinaxNOW testing and Bio-Plex testing performed, and a total of 1004 (88.8\%) individuals had blood cultures performed following admission.

Pneumococcal aetiology was defined as a positive result in any of the following: blood culture, BinaxNOW or Bio-Plex. Serotypes contained within the seven-valent childhood conjugate vaccine $(4,6 \mathrm{~B}, 9 \mathrm{~V}, 14,18 \mathrm{C}, 19 \mathrm{~F}, 23 \mathrm{~F})$ were defined as PCV7 serotypes and others as non-PCV7 serotypes.
Statistical analysis was performed using SPSS V.20.0. Pearson's $\chi^{2}$ test was used to compare categorical variables and generate odd ratios and 95\% CIs. Mann-Whitney U test was used to compare continuous variables. Age was grouped into the following categories: $16-49,50-64,65-74,75-85$ and $85+$ years. Multivariate logistical regression analysis was used to investigate the association between pneumococcal disease and child contact. The following variables were considered as potential confounders: age, sex, individual comorbidities (malignancy, congestive cardiac failure, chronic kidney disease, liver disease, stroke disease, asthma, chronic obstructive pulmonary disease, diabetes, dementia and HIV), adult plain pneumococcal polysaccharide vaccination status and smoking status. ${ }^{112122}$ Variables that led to a greater than $10 \%$ change in the magnitude of the regression coefficient of the association between child contact and pneumococcal aetiology were retained in the final model. A 'positive smoking history' defined current smokers or ex-smokers who had used over a 100 cigarettes in their lifetime and 'never smokers' were defined as patients who had smoked less than 100 cigarettes in their lifetime. ${ }^{11}$ Given higher rates of nasopharyngeal carriage in children aged $\leq 5$ years, a subgroup analysis of adults who had contact with preschool age children ( $\leq 5$ years old) was performed to investigate any association with pneumococcal CAP and PCV7 serotype disease.

All patients were managed at the discretion of the admitting clinical team according to local CAP guidelines. Ethics approval was granted by the Nottingham Regional Ethics Committee.

\section{RESULTS}

A total of 1166 patients were eligible for inclusion in the study. Child contact data were not available in 36 patients, leaving 1130 for analysis. Median age of the cohort was 71.0 years (IQR 57.0-80.1 years) and 58.1\% were men. Overall 30-day mortality was $9.6 \%$ and median length of stay 6.3 days (IQR 3.1-12.3). Child contact was reported in 329 of 1130 (29.1\%) individuals; the median age for child contacts was 4.0 years (IQR 2.0-7.0).

Pneumococcal aetiology was identified in 410 of 1130 $(36.3 \%)$ individuals; BinaxNOW urinary antigen test was positive in 237 of $410(57.8 \%)$ individuals, blood culture was positive in $43(10.5 \%)$ and Bio-Plex assay was positive in 274 $(66.8 \%)$. The microbial aetiology of the entire cohort is described in online supplementary table S1.

\section{Clinical features of adults according to child contact status}

The child contact group was associated with younger age, female sex, and less adult plain polysaccharide pneumococcal vaccination on univariate analysis (table 1). Comorbidities including malignancy, congestive cardiac failure, stroke disease, chronic kidney disease, chronic obstructive pulmonary disease and dementia were less common in the child contact group. However, asthma was commoner in this group and individuals were more likely to have a positive smoking history.

Pneumonia severity, stratified according to pneumonia severity index (PSI) and CURB-65 risk classes (confusion, urea $>7 \mathrm{mmol} / \mathrm{L}$, respiratory rate $\geq 30 / \mathrm{min}$, systolic blood pressure $<90 \mathrm{~mm} \mathrm{Hg}$ or diastolic blood pressure $\leq 60 \mathrm{~mm} \mathrm{Hg}$ and age $\geq 65$ years), was significantly lower in the group with child contact ( $\mathrm{p}$ value for trend $<0.001$ for both) (table 1). Thirty-day mortality was also significantly lower in the group with child contact $(4.9 \%$ vs $11.5 \%$ following adjustment for the PSI (OR 0.52, CI 0.29 to $0.94 ; \mathrm{p}=0.029$ ). Median length of stay unadjusted for disease severity was longer in the group with 
Table 1 Clinical characteristics of individuals according to child contact status

\begin{tabular}{|c|c|c|c|c|c|}
\hline & $\begin{array}{l}\text { Child contact } \\
(n=329)\end{array}$ & $\begin{array}{l}\text { No child contact } \\
(n=801)\end{array}$ & OR & $95 \% \mathrm{Cl}$ & p Value \\
\hline $\mathrm{Age}^{*}$ & $62.4(43.2-74.3)$ & $73.0(61.1-82.1)$ & & & $<0.001$ \\
\hline Male sex & $162(49.2)$ & $494(61.7)$ & 0.60 & 0.46 to 0.78 & $<0.001$ \\
\hline Positive smoking history & $242(73.6)$ & $536(66.9)$ & 1.38 & 1.03 to 1.83 & 0.029 \\
\hline PPV & $114(34.7)$ & $357(44.6)$ & 0.60 & 0.45 to 0.78 & $<0.001$ \\
\hline Malignancy & $15(4.6)$ & $67(8.4)$ & 0.52 & 0.29 to 0.93 & 0.025 \\
\hline Liver disease & $3(0.9)$ & $10(1.2)$ & 0.73 & 0.20 to 2.66 & 0.630 \\
\hline CCF & $15(4.6)$ & $65(8.1)$ & 0.54 & 0.30 to 0.96 & 0.033 \\
\hline Stroke disease & $26(7.9)$ & $97(12.1)$ & 0.62 & 0.40 to 0.98 & 0.039 \\
\hline Chronic kidney disease & $10(3.0)$ & $63(7.9)$ & 0.37 & 0.18 to 0.72 & 0.003 \\
\hline Asthma & $45(13.7)$ & $77(9.6)$ & 1.49 & 1.01 to 2.21 & 0.045 \\
\hline COPD & 39 (11.9) & $155(19.4)$ & 0.56 & 0.38 to 0.82 & 0.002 \\
\hline Diabetes & $37(11.2)$ & $124(15.5)$ & 0.69 & 0.47 to 1.02 & 0.064 \\
\hline Dementia & $5(1.5)$ & $33(4.1)$ & 0.36 & 0.14 to 0.93 & 0.028 \\
\hline HIV & $1(0.3)$ & $4(0.5)$ & 0.61 & 0.07 to 5.45 & 0.653 \\
\hline PSI risk class I-III & $219(67.2)$ & $346(43.2)$ & & & $<0.001 \dagger$ \\
\hline PSI risk class IV & $76(23.2)$ & $320(40.0)$ & & & \\
\hline PSI risk class V & $31(9.5)$ & $134(16.8)$ & & & \\
\hline CURB65 0-1 & $194(59.5)$ & 309 (38.9) & & & $<0.001 t$ \\
\hline CURB65 2 & $82(24.9)$ & $279(34.8)$ & & & \\
\hline CURB65 3-5 & $50(15.3)$ & $207(26.0)$ & & & \\
\hline Pneumococcal disease & $148(45.0)$ & $262(32.7)$ & 1.68 & 1.29 to 2.18 & $<0.001$ \\
\hline
\end{tabular}

no child contact (6.7 days (IQR 3.2-13.3) vs 5.2 days (IQR 3.09.8), respectively, $\mathrm{p}=0.006)$.

\section{The association of pneumococcal aetiology with child contact}

The odds of pneumococcal pneumonia were significantly greater in the group with child contact (148 of $329(45.0 \%)$ vs 262 of 801 (32.7\%); OR 1.68, CI 1.29 to $2.18 ; \mathrm{p}<0.001)$ (table 1). This association was maintained on multivariate analysis (OR 1.63 , CI 1.25 to 2.14 ; $\mathrm{p}<0.001$ ) (table 3 ).

Malignancy was less common and individuals were more likely to be women in the group with pneumococcal CAP (table 2). In addition to child contact, age and sex were independently associated with pneumococcal CAP (table 3). Eight of the 16 individuals who were in employment that involved full-time contact with children had pneumococcal CAP.

The odds of pneumococcal CAP did not differ between adults who had contact with a vaccinated child versus those in contact with an unvaccinated child (47 of $92(51.1 \%)$ in the group with a vaccinated contact versus 101 of 237 (42.6\%) in the group with an unvaccinated contact; OR $1.42,95 \%$ CI 0.87 to 2.33 ; $\mathrm{p}=0.157)$.

\section{Association of pneumococcal serotypes with vaccination status of child contacts}

A serotype was determined in 274 of 410 (66.8\%) adults with pneumococcal aetiology. Three of these adults simultaneously had PCV7 and non-PCV7 serotypes identified (serotype 4 and 8 ); all of them had non-invasive CAP. The distribution of pneumococcal serotypes by child contact status is illustrated in online supplementary table S2. Of the eight individuals in employment involving full-time contact with children, six had CAP due to a non-PCV7 serotype (four adults with serotype 1, one adult each with serotype 8 and 19A) and two had a PCV7 serotype (serotype 14 and $9 \mathrm{~V}$ ); it was not possible to ascertain vaccination status of all of their child contacts. Of the remaining 263 adults, $112(42.6 \%)$ reported contact with a child, of whom 38 (33.9\%) reported contact with a vaccinated child (table 4). Vaccine uptake amongst eligible child contacts was high (38 of $42(90.5 \%)$ children). All vaccinated child contacts in this study received at least one dose of PCV7 and no PCV13 doses by the admission date of the corresponding adult with CAP. The background PCV coverage for the Nottingham City Primary Care Trust area was as follows: $82.0 \%$ in $2008 ; 90.4 \%$ in 2009; $91.3 \%$ in 2010; and $92.4 \%$ in $2011^{23}$

Adults who had contact with a vaccinated child were significantly less likely to have CAP due to a PCV7 serotype compared to adults who had contact with an unvaccinated child (6 of 38 $(15.8 \%)$ vs 25 of $74(33.8 \%)$ respectively, OR 0.37 , 95\% CI 0.14 to $0.99 ; p=0.044$ ) (table 4 ) (figure 1). Serotype 1 was the most prevalent non-PCV7 serotype in the vaccinated contact group, and serotype 14 was the most prevalent PCV7 serotype in the unvaccinated child contact group.

In the subgroup analysis of adults who had contact with children aged $\leq 5$ years compared with adults who had contact with older children or with no child contact, there was a trend for pneumococcal aetiology to be commoner in the former (104 of $251(41.4 \%)$ vs 306 of 879 (34.8\%), respectively; OR 1.30, $95 \%$ CI 0.98 to $1.74 ; \mathrm{p}=0.298)$. Similarly, there was a trend towards lower odds of PCV7 serotype CAP in adults who had contact with a vaccinated child $\leq 5$ years in comparison to unvaccinated children within this age group (6 of $37(16.2 \%)$ vs 
Table 2 Putative risk factors for pneumococcal CAP following univariate analysis

\begin{tabular}{|c|c|c|c|c|c|}
\hline & Pneumococcal CAP $(n=410)$ & Non-pneumococcal CAP $(n=720)$ & OR & $95 \% \mathrm{Cl}$ & p Value \\
\hline Age $^{*}$ & $70.0(50.0-80.1)$ & $71.6(59.0-80.3)$ & & & 0.091 \\
\hline Male sex & $217(52.9)$ & $434(60.3)$ & 0.72 & 0.56 to 0.92 & 0.008 \\
\hline Positive smoking history & $289(70.5)$ & $481(66.8)$ & 1.14 & 0.88 to 1.49 & 0.324 \\
\hline PPV & $160(39.0)$ & $307(42.6)$ & 0.90 & 0.69 to 1.16 & 0.410 \\
\hline Malignancy & $21(5.1)$ & $61(8.4)$ & 0.58 & 0.34 to 0.96 & 0.032 \\
\hline Liver disease & $8(2.0)$ & $5(0.69)$ & 2.81 & 0.91 to 8.65 & 0.060 \\
\hline CCF & $28(6.8)$ & $52(7.2)$ & 0.93 & 0.58 to 1.50 & 0.773 \\
\hline Stroke disease & $48(11.7)$ & $75(10.4)$ & 1.12 & 0.76 to 1.65 & 0.550 \\
\hline Chronic kidney disease & $29(7.1)$ & $44(6.1)$ & 1.16 & 0.71 to 1.88 & 0.553 \\
\hline Asthma & $54(13.1)$ & $67(9.3)$ & 1.46 & 1.00 to 2.13 & 0.050 \\
\hline COPD & $62(15.1)$ & 129 (17.9) & 0.80 & 0.58 to 1.12 & 0.195 \\
\hline Diabetes & 53 (12.9) & $104(14.4)$ & 0.87 & 0.61 to 1.24 & 0.429 \\
\hline Dementia & $18(4.4)$ & $20(2.8)$ & 1.59 & 0.83 to 3.03 & 0.160 \\
\hline HIV & $2(0.5)$ & $3(0.4)$ & 1.16 & 0.19 to 6.95 & 0.873 \\
\hline Child contact & $148(36.1)$ & $181(25.1)$ & 1.68 & 1.29 to 2.18 & $<0.001$ \\
\hline
\end{tabular}

Bold values represent $p$ values reaching statistical significance $(<0.05)$.

All values given as $\mathrm{n}(\%)$ unless stated otherwise.

${ }^{*}$ Median years (IQR).

CAP, community acquired pneumonia; CCF, congestive cardiac failure; COPD, chronic obstructive pulmonary disease; PPV, pneumococcal polysaccharide vaccination.

12 of 35 (34.3\%), respectively; OR $0.37,95 \%$ CI 0.12 to 1.13 ; $\mathrm{p}=0.077)$.

\section{DISCUSSION}

The main findings of this study are that adults who are in contact with a child have a higher chance of CAP due to $S$ pneumoniae, and PCV7 serotypes featured less in adults with CAP who were in contact with a PCV7-vaccinated child.

The impact of child contact on the risk of developing IPD has been investigated in the past in children and adults. Outbreaks of IPD have been described amongst children living in close contact in childcare homes, ${ }^{24}$ and daycare attendance has been shown to increase the risk of IPD in young children in casecontrol studies. ${ }^{10} \mathrm{~A}$ study by Nuorti $e t a l^{11}$ investigating the effects of smoking and IPD, found contact with a child less than 6 years of age attending daycare to be an independent risk factor for the development of IPD in adults. A case-control study investigating the efficacy of pneumococcal polysaccharide vaccination in patients with HIV infection by Breiman and colleagues found child contact to be independently associated with a $90 \%$ higher risk of IPD. ${ }^{12}$

Studies of IPD and CAP in the years following introduction of child pneumococcal vaccination provide further indirect evidence of the link between child contact and adult disease; cases of IPD identified through Active Bacterial Core Surveillance sites in the Unites States have shown a reduction in rates of adult disease in the years following childhood pneumococcal

Table 3 Associations with pneumococcal CAP following multivariate analysis

\begin{tabular}{lllr}
\hline & Adjusted OR & 95\% Cl & p Value \\
\hline Age & - & - & $\mathbf{0 . 0 0 2}$ \\
Male sex & 0.77 & 0.60 to 0.99 & $\mathbf{0 . 0 4 1}$ \\
Child contact & 1.63 & 1.25 to 2.14 & $<0.001$ \\
\hline Bold values represent $p$ values reaching statistical significance $(<0.05)$. & \\
OR adjusted for potential confounders for pneumococcal disease. \\
CAP, community acquired pneumonia.
\end{tabular}

vaccination, ${ }^{13} 14$ while large database analyses have noted a decline in pneumonia admissions in all age groups following introduction of childhood vaccination. ${ }^{25} \mathrm{In}$ addition, spikes in annual pneumococcal disease rates in adults, which appeared to coincide with winter holiday periods when close social contact with children was likely to occur, have largely been eliminated following introduction of the childhood pneumococcal vaccine. ${ }^{26}$

To our knowledge, this is the first study to investigate the association between child contact and non-IPD in adults with evidence of herd protection at an individual level, rather than a population level.

Children are thought to act as a reservoir for the spread of pneumococcal disease within the community owing to the high rates of nasopharyngeal colonisation in childhood. $^{7}{ }^{8}$ Homologous transmission of pneumococcal serotypes has been demonstrated amongst children and adults living within the same household. ${ }^{9} 27$ Childhood pneumococcal conjugate vaccination appears to reduce the rate of nasopharyngeal carriage of vaccinetype pneumococcal serotypes in children ${ }^{28} 29$ with consequent diminished carriage of similar serotypes in adults living in close contact with vaccinated children. ${ }^{30}$ The current study provides new data demonstrating the important relationship between childhood vaccination, child contact and adult pneumonia.

Apart from serotype 19F, the number of cases of PCV7-type disease was lower in the group that had contact with vaccinated children compared with unvaccinated children (table 4). The numbers with serotype $19 \mathrm{~F}$ disease were small. A relevant finding from studies of pneumococcal conjugate vaccine efficacy in children is of vaccine failures and lower efficacy against acute otitis media and pneumococcal disease due to serotype $19 \mathrm{~F}^{31}{ }^{32}$ Relative differences in the ability of serotype $19 \mathrm{~F}$ to resist complement deposition and the requirement for higher antibody titres for opsonophagocytic killing may explain these observations. ${ }^{33}$ A relative failure of vaccination to clear or prevent nasopharyngeal carriage of serotype $19 \mathrm{~F}$ in children might in turn result in a lack of protection of adults from serotype $19 \mathrm{~F}$ disease.

In contrast to previous studies of IPD, ${ }^{11}{ }^{21}$ female sex and younger age were independently associated with pneumococcal CAP in our cohort of patients with predominantly non-invasive 
Table 4 Distribution of pneumococcal serotypes between vaccinated child contact versus unvaccinated child contact groups

\begin{tabular}{|c|c|c|c|c|c|c|c|c|c|c|c|c|c|c|c|c|c|c|c|}
\hline & \multicolumn{7}{|c|}{ PCV7 serotypes ( $n=31)$} & \multirow[b]{2}{*}{ Total n (\%) } & \multicolumn{10}{|c|}{ Non-PCV7 serotypes $(n=81)$} & \multirow[b]{2}{*}{ Total n (\%) } \\
\hline & 4 & $6 B$ & $9 \mathrm{~V}$ & 14 & 18 & $19 \mathrm{~F}$ & $6 B+9 V$ & & 1 & 3 & 5 & $6 \mathrm{~A} / \mathrm{C}$ & 7F/A & 8 & $19 \mathrm{~A}$ & $22 \mathrm{~F}$ & $33 F$ & $35 B$ & \\
\hline Contact with unvaccinated child $(n=74)$ & 5 & 1 & 2 & 13 & 3 & 0 & 1 & $25(33.8)$ & 11 & 2 & 1 & 6 & 9 & 12 & 5 & 1 & 1 & 1 & $49(66.2)$ \\
\hline Contact with vaccinated child $(n=38)$ & 1 & 0 & 0 & 3 & 0 & 2 & 0 & $6(15.8)$ & 13 & 2 & 3 & 0 & 5 & 4 & 5 & 0 & 0 & 0 & $32(84.2)$ \\
\hline
\end{tabular}

PCV7, seven-valent pneumococcal conjugate vaccine; non-PCV7, non-vaccine type.

disease. Other putative risk factors based on studies of bacteraemic pneumococcal disease were not significantly associated with pneumococcal CAP in our cohort. ${ }^{11}{ }^{21}$ Although this study was based on a large cohort of patients with CAP, the number of patients with dementia and HIV were relatively small; hence results relating to such patients should be interpreted with caution.

Obtaining a history of recent child contact is prone to recall bias. To minimise this, data obtained from any patients or their relatives that were inconsistent or inaccurate were excluded from analyses. The sensitivity of the Bio-Plex assay in determining pneumococcal serotype is $79 \%,{ }^{20}$ and a proportion of pneumococcal disease would have been missed. However, this would underestimate the true differences between the groups with pneumococcal and non-pneumococcal aetiology, including child contact. As the Bio-Plex assay detects only 14 serotypes, some non-PCV7 serotypes may have been missed; this may have led to an underestimate of the true differences between the groups with vaccinated or unvaccinated child contact. The overall specificity of the BinaxNOW test for pneumococcal pneumonia is $>97 \%{ }^{34}$; however, specificity may be lower $(\sim 92 \%)$ in adults with chronic obstructive pulmonary disease (COPD) due to pneumococcal colonisation. ${ }^{35}$ In this study, patients with COPD all had radiological evidence of CAP. In addition, as more patients with COPD had no child contact, any decreased specificity would mean the observed differences are conservative.

Population-level herd protection effects following introduction of PCV13 to the UK immunisation schedule in April 2010

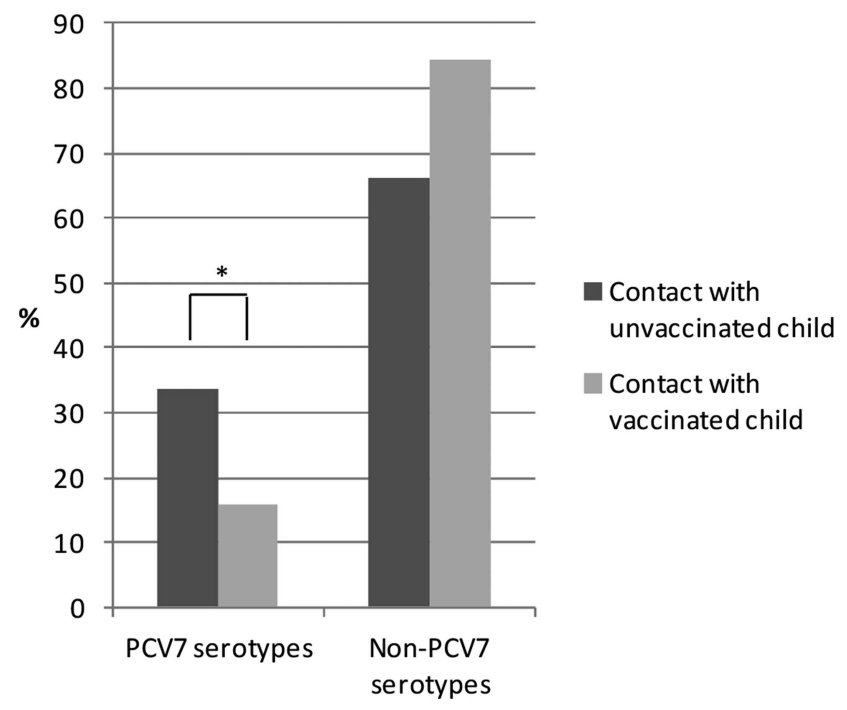

Figure 1 Proportion of PCV7 and non-PCV7 serotypes in adults with vaccinated child contact versus unvaccinated child contact groups. PCV7, seven-valent pneumococcal conjugate vaccine; non-PCV7, non-vaccine-type. ${ }^{*} p=0.044$. may have reduced the proportion of non-PCV7 serotype CAP in adults recruited towards the latter part of this study; this would have affected 'child contact' and 'no child contact' groups equally in this study as none of the vaccinated child contacts had received PCV13 vaccination. Further studies to determine the pneumococcal serotypes associated with nasopharyngeal colonisation in child contacts would provide useful data on the relationships described. The challenges associated with such a study would include the interpretation of the role of any serotypes detected taking into account the possibility of multiple colonising serotypes and the loss or acquisition of new serotypes over time.

In conclusion, child contact is associated with pneumococcal infection in adults with CAP. The mechanism underlying this is likely to be acquisition of $S$ pneumoniae from children colonised with similar pneumococcal serotypes through horizontal transmission. The epidemiology of adult CAP will continue to change as herd protection effects due to PCV13 (and other putative higher valency conjugate pneumococcal vaccines) become manifest. Continued serotype-specific surveillance of invasive and non-invasive adult CAP is warranted.

Acknowledgements The authors would like to thank Tim Harrison from RSIL, Colindale for his firm support; Christine More and Seyi Elutu from RSIL, Colindale for processing the urine samples using the Bio-Plex assay; Robert Cave, Andrew Shelton, Adrian Patrick, Michelle Stannard and Joanne Palfreyman from the Nottingham University Hospitals Microbiology Department for processing the urine specimens using the BinaxNOW assay; clinicians and staff of Nottingham University Hospitals NHS Trust, and Gemma Thompson, Emily Jarvis, Melanie Caine and Gaynor Bates for assisting with patient recruitment. The authors acknowledge the support of Alere in providing BinaxNOW test kits towards the conduct of this study.

Contributors All authors participated in study design; CR, TB and SG recruited study subjects; CS conducted study procedures; VM provided child contact data; CR and WSL analysed and interpreted the results; CT provided statistical expertise; CR drafted the article; all authors reviewed and revised the article prior to final approval.

Funding This report is independent research arising from a Biomedical Research Fellowship supported by the National Institute for Health Research (NIHR), and an unrestricted grant from Pfizer.

Competing interests $C R$ has received salaries part funded by an unrestricted grant from Pfizer and NIHR grant. TB and SG have received salaries derived from an unrestricted grant from Pfizer. VM has no competing interests to declare. CS has received support for travel to meetings for other purposes from Pfizer. MS has served on advisory boards for Pfizer, GlaxoSmithKline (GSK) and Merck, and her department has received research grants from Pfizer and GSK, and has received payment for lectures at symposia in overseas conferences organised by GSK and Pfizer. RG has received grants from Pfizer and GSK, and has received travel and accommodation expenses for overseas conferences from Pfizer and GSK. WSL has received an unrestricted research grant from Pfizer.

Ethics approval The study was approved by the Nottingham regional ethics committee.

Provenance and peer review Not commissioned; externally peer reviewed.

\section{REFERENCES}

1 Trotter CL, Stuart JM, George R, et al. Increasing hospital admissions for pneumonia, England. Emerg Infect Dis 2008;14:727-33. 
2 Rodrigo C, Mckeever TM, Woodhead M, et al. Single versus combination antibiotic therapy in adults hospitalised with community acquired pneumonia. Thorax 2013:68:493-5.

3 Menendez R, Torres A, Zalacain R, et al. Risk factors of treatment failure in community acquired pneumonia: implications for disease outcome. Thorax 2004;59:960-65

4 Welte T, Torres A, Nathwani D. Clinical and economic burden of community-acquired pneumonia among adults in Europe. Thorax 2012;67:71-9.

5 Cillóniz C, Ewig S, Polverino E, et al. Microbial aetiology of community-acquired pneumonia and its relation to severity. Thorax 2011;66:340-6.

6 Bogaert D, de Groot R, Hermans PWM. Streptococcus pneumoniae colonisation: the key to pneumococcal disease. Lancet Infect Dis 2004:4:144-54.

7 Regev-Yochay G, Dagan R, Raz M, et al. Association between carriage of Streptococcus pneumoniae and Staphylococcus aureus in children. JAMA 2004;292:716-20.

8 Hussain M, Melegaro A, Pebody RG, et al. A longitudinal household study of Streptococcus pneumoniae nasopharyngeal carriage in a UK setting. Epidemiol Infect 2005;133:891-8

9 Hill PC, Townend J, Antonio M, et al. Transmission of Streptococcus pneumoniae in rural Gambian villages: a longitudinal study. Clin Infect Dis 2010;50:1468-76.

10 Levine OS, Farley M, Harrison LH, et al. Risk factors for invasive pneumococcal disease in children: a population-based case-control study in North America. Pediatrics 1999:103:e28.

11 Nuorti JP, Butler JC, Farley MM, et al. Cigarette smoking and invasive pneumococcal disease. Active Bacterial Core Surveillance Team. N Engl J Med 2000;342:681-9.

12 Breiman RF, Keller DW, Phelan MA, et al. Evaluation of effectiveness of the 23-valent pneumococcal capsular polysaccharide vaccine for HIV-infected patients. Arch Intern Med 2000;160:2633-8.

13 Pilishvili T, Lexau C, Farley MM, et al. Sustained reductions in invasive pneumococcal disease in the era of conjugate vaccine. $J$ Infect Dis 2010;201:32-41.

14 Whitney CG, Farley MM, Hadler J, et al. Decline in invasive pneumococcal disease after the introduction of protein-polysaccharide conjugate vaccine. $N$ Engl J Med 2003;348:1737-46

15 Lexau CA, Lynfield R, Danila $R$, et al. Changing epidemiology of invasive pneumococcal disease among older adults in the era of pediatric pneumococcal conjugate vaccine. JAMA 2005;294:2043-51.

16 Metlay JP, Fishman NO, Joffe M, et al. Impact of pediatric vaccination with pneumococcal conjugate vaccine on the risk of bacteremic pneumococcal pneumonia in adults. Vaccine 2006:24:468-75.

17 Grijalva CG, Nuorti JP, Arbogast PG, et al. Decline in pneumonia admissions after routine childhood immunisation with pneumococcal conjugate vaccine in the USA: a time-series analysis. Lancet 2007;369:1179-86.

18 Nelson JC, Jackson M, Yu 0, et al. Impact of the introduction of pneumococcal conjugate vaccine on rates of community acquired pneumonia in children and adults. Vaccine 2008:26:4947-54.
19 Kristiansen BE, Tveten Y, Jenkins A. Which contacts of patients with meningococcal disease carry the pathogenic strain of Neisseria meningitidis? A population based study. BMJ 1998;317:621-5.

20 Sheppard CL, Harrison TG, Smith MD, et al. Development of a sensitive, multiplexed immunoassay using XMAP beads for detection of serotype-specific Streptococcus pneumoniae antigen in urine samples. J Med Microbiol 2011;60:49-55.

21 Centers for Disease Control and Prevention. Prevention of pneumococcal disease: recommendations of the Advisory Committee on Immunization Practices (ACIP). MMWR Recomm Rep 1997;46(RR-8):1-24.

22 Moberley SA, Holden J, Tatham DP, et al. Vaccines for preventing pneumococcal infection in adults. Cochrane Database Syst Rev 2008;(1):CD000422.

23 Public Health England. Vaccine coverage data. http://www.hpa.org.uk/webw/ HPAweb\&HPAwebStandard/HPAweb_C/12114414422882013 (cited Aug 2013).

24 Cherian T, Steinhoff MC, Harrison LH, et al. A cluster of invasive pneumococcal disease in young children in child care. JAMA 1994;271:695-7.

25 Simonsen L, Taylor RJ, Young-Xu Y, et al. Impact of pneumococcal conjugate vaccination of infants on pneumonia and influenza hospitalization and mortality in all age groups in the United States. mBio 2011;2:e00309-10.

26 Walter ND, Taylor TH, Dowell SF, et al. Holiday spikes in pneumococcal disease among older adults. N Engl J Med 2009;361:2584-5.

27 Reis JN, Palma T, Ribeiro GS, et al. Transmission of Streptococcus pneumoniae in an urban slum community. J Infect 2008;57:204-13.

28 Huang SS, Hinrichsen VL, Stevenson AE, et al. Continued impact of pneumococcal conjugate vaccine on carriage in young children. Pediatrics 2009;124:e1-e11.

29 Pelton SI, Loughlin AM, Marchant CD. Seven valent pneumococcal conjugate vaccine immunization in two Boston communities: changes in serotypes and antimicrobial susceptibility among Streptococcus pneumoniae isolates. Pediatr Infect Dis J 2004:23:1015-22.

30 Millar EV, Watt JP, Bronsdon MA, et al. Indirect effect of 7-valent pneumococcal conjugate vaccine on pneumococcal colonization among unvaccinated household members. Clin Infect Dis 2008;47:989-96.

31 Black S, Shinefield $H$, Fireman B, et al. Efficacy, safety and immunogenicity of heptavalent pneumococcal conjugate vaccine in children. Northern California Kaiser Permanente Vaccine Study Center Group. Pediatr Infect Dis J 2000;19:187-95.

32 Whitney CG, Pilishvili T, Farley MM, et al. Effectiveness of seven-valent pneumococcal conjugate vaccine against invasive pneumococcal disease: a matched case-control study. Lancet 2006;368:1495-502.

33 Melin M, Jarva H, Siira L, et al. Streptococcus pneumoniae capsular serotype $19 \mathrm{~F}$ is more resistant to $\mathrm{C} 3$ deposition and less sensitive to opsonophagocytosis than serotype 6B. Infect Immun 2009;77:676-84.

34 Sinclair A, Xie X, Teltscher $M$, et al. Systematic review and meta-analysis of a urine-based pneumococcal antigen test for diagnosis of community-acquired pneumonia caused by Streptococcus pneumoniae. J Clin Microbiol 2013;51:2303-10.

35 Briones ML, Blanquer J, Ferrando D, et al. Assessment of analysis of urinary pneumococcal antigen by immunochromatography for etiologic diagnosis of community-acquired pneumonia in adults. Clin Vaccine Immunol 2006;13:1092-7. 